BMJ Paediatrics Open

\title{
Prospective study of 5-day challenge with penicillins in children
}

\author{
Birgitte Tusgaard Petersen, ${ }^{1}$ Josefine Gradman (i) ${ }^{2,3}$
}

\begin{abstract}
To cite: Petersen BT, Gradman J. Prospective study of 5-day challenge with penicillins in children. BMJ Paediatrics Open 2020:4:e000734. doi:10.1136/ bmjpo-2020-000734
\end{abstract}

Received 16 May 2020 Revised 2 July 2020 Accepted 6 July 2020

\section{Check for updates}

(c) Author(s) (or their employer(s)) 2020. Re-use permitted under CC BY-NC. No commercial re-use. See rights and permissions. Published by BMJ.

${ }^{1}$ Department of Paediatrics, Regional Hospital Central Jutland, Viborg, Denmark ${ }^{2}$ Hans Christian Andersen Children's Hospital, Odense University Hospital, Odense, Denmark

${ }^{3}$ OPEN, Odense Patient Data Explorative Network, Odense University Hospital, Odense, Denmark

Correspondence to Dr Josefine Gradman; josefine. gradman@rsyd.dk

\section{ABSTRACT}

Objectives To examine if a 5-day challenge with penicillin improves the diagnostic sensitivity compared with a single full dose in children with mild skin reactions. Design Subjects referred with suspected allergy to penicillin were consecutively included. Irrespectively of the morphology of the index reaction and the result of specific IgE, all subjects underwent a two-step titrated drug provocation test (DPT) with the culprit drug followed by a 5 -day challenge at home.

Participants Children and adolescents aged $0-18$ years referred to allergic workup for penicillin hypersensitivity at two paediatric Danish centres. Only subjects with nonsevere skin reactions were included.

Results A total of 305 subjects were included and 22 $(7 \%)$ of the DPTs were positive. Three subjects reacted within 1 hour of the first full dose and nine reacted 1-8 hours after the first full dose. Additional 10 positive reactions were observed during the prolonged provocation. Seven subjects reacted after the second full dose and three reacted after 3-6 days. Only mild skin rashes were observed. Eighteen subjects had a specific lgE to a penicillin $>0.1 \mathrm{kU} / \mathrm{L}$. Only one of these had a positive DPT. Conclusion In children, a DPT with penicillins should include at least two full doses. In children with mild hypersensitivity reactions it may be safe to perform DPTs despite a low specific lgE.

Trial registration number NCT04331522

\section{INTRODUCTION}

Parent-reported prevalence of penicillin allergy in children is around $10 \%$, but the real prevalence is considerably lower. ${ }^{1}{ }^{2}$ An erroneous penicillin allergy labelling leads to inappropriate prescription of broad spectrum antibiotics that are more expensive, may be less effective and often have more side effects than penicillins. ${ }^{3}$ The optimal protocol for evaluating hypersensitivity reactions to penicillins in children is debated, and a recent survey illustrated significant heterogeneity in the diagnostic approach to $\beta$-lactam hypersensitivity. ${ }^{4}$

The European Network for Drug Allergy (ENDA), European Academy of Allergology and Clinical Immunology (EAACI) has recommended separate diagnostic algorithms for immediate and for non-immediate hypersensitivity reactions to $\beta$-lactams. ${ }^{56}$ The distinction between the two types of reaction
What is known about the subject?

- The vast majority of drug provocation tests with penicillins in children are negative. To perform prolonged provocations may pick up additional allergic reactions.

What this study adds?

- This study illustrates the value of prolonged provocation tests.

- Only $55 \%$ of the positive reactions appeared before the second full dose was administered.

- Interestingly, a specific lgE value between $0.1 \mathrm{kU} / \mathrm{L}$ and $1.01 \mathrm{kU} / \mathrm{L}$ was not associated with a positive provocation.

was previously based exclusively on the time interval from drug intake to the onset of a reaction, that is, immediate reactions appearing within 1 hour, and non-immediate reactions appearing more than 1 hour after drug intake. ${ }^{7}$ In a recently published updated guideline from the EAACI Drug Allergy Interest Group (DAIG), a new classification of hypersensitivity reactions to $\beta$-lactams was introduced. This novel classification is based on both the chronology and the morphology of the index reaction with the time intervals overlapping. ${ }^{8}$ An immediate reaction, typically urticaria, may occur up to 6 hours after the last administered dose, while nonimmediate reactions can occur from 1 hour to several days after the initial drug administration and can present as maculopapular rash or delayed urticaria. The diagnostic workup should be differentiated, based on a risk stratification of the index reaction. The authors, however, conclude that further studies are needed to provide data supporting the standardisation of drug provocation test (DPT) protocols. Particularly, consensus is lacking concerning the optimal dosing schedules and whether extended DPTs are needed. In addition, it may be advisable to have separate protocols for children and adults. 
In the present study performed at two Danish paediatric departments, we prospectively included children referred with a history of mild hypersensitivity reaction to oral penicillin. The same DPT protocol was used for all subjects irrespectively of the classification of the index reaction and the result of specific IgE.

The purpose of the study was to evaluate whether a prolonged DPT at home for 5 days picks up additional positive reactions compared with a two-step provocation test with a single therapeutic dose. In addition, we wanted to evaluate the ability of skin prick test (SPT), specific IgE and the morphology of the index reaction to predict the outcome of the DPT.

\section{METHODS}

\section{Subjects}

During a 5-year period from 2014 to 2019, all children aged $0-18$ years with suspected allergy to penicillins were successively included in the study. The children were referred to the local paediatric department, depending on place of residence, the Regional Hospital Central Jutland or the Hans Christian Andersen Children's Hospital, Odense University Hospital. All included children had either developed a skin rash during oral treatment with penicillin leading to discontinuation of the drug or they had developed a rash within 48 hours after ended penicillin treatment. Subjects with a history of anaphylaxis and children with severe non-immediate reactions (Stevens-Johnson syndrome, toxic epidermal necrolysis and acute generalised exanthematous pustulosis) were excluded.

At both centres the allergy workup and the DPTs were supervised by a paediatric allergologist.

\section{Allergy workup}

Prior to the DPT, the index reaction was classified based on history, pictures on cell phones and medical records. Specific IgE (ImmunoCAP, Thermo-Fisher Scientific, Uppsala, Sweden) to benzylpenicillin, penicillin G, amoxicillin and ampicillin was measured with a cut-off value of $0.1 \mathrm{kU} / \mathrm{L}$. At the university hospital, only, an SPT with the undiluted culprit drug was performed immediately prior to the DPT. ${ }^{8}$ In case the culprit drug was amoxicillinclavulanic acid, the SPT was performed with amoxicillin alone. Reactions to SPT were considered positive when the diameter of the weal was at least $3 \mathrm{~mm}$ compared with the negative control (saline).

\section{Drug provocation test}

All children and adolescents underwent an open oral DPT with the culprit drug. The in-hospital part of the DPT was performed in two steps: an initial dose of one tenth of a therapeutic dose and subsequently after 1 hour a full therapeutic dose according to weight. The used therapeutic doses were: phenoxymethylpenicillin: $15 \mathrm{mg} / \mathrm{kg}$ maximum $800 \mathrm{mg}$, amoxicillin: $15 \mathrm{mg} / \mathrm{kg}$ maximum $500 \mathrm{mg}$, dicloxacillin: $15 \mathrm{mg} / \mathrm{kg}$ maximum $1000 \mathrm{mg}$ according to the

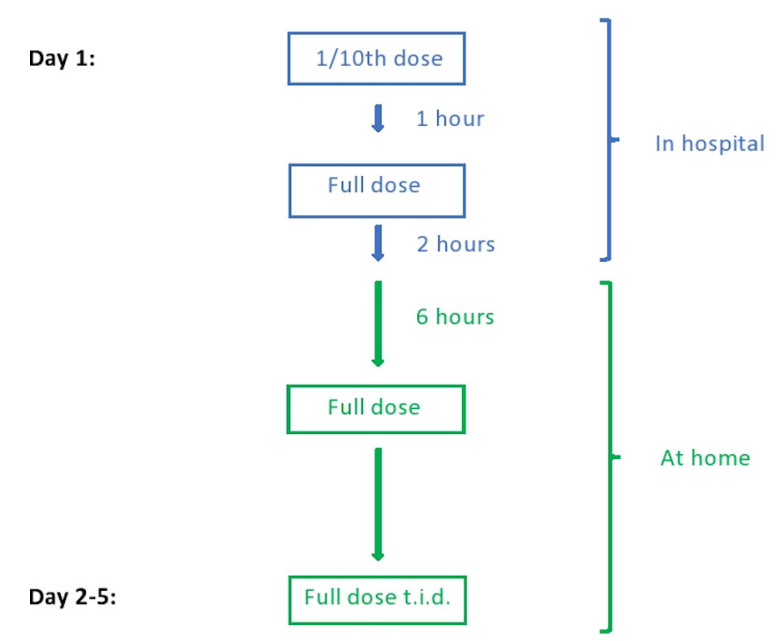

Figure 1 A schematic illustration of the drug provocation test. t.i.d, three times a day.

Danish summaries of product characteristics. ${ }^{9}$ The children were observed for 2 hours at the department and then continued with a 5 -day provocation at home (figure 1). The first dose at home was administered in the evening, approximately 8 hours after the first full dose. The following 4 days, a therapeutic dose was administered three times a day. The DPT was considered positive if any objective allergic symptoms occurred during the DPT or the following 48 hours. The subjects were provided with the drug, a medication schedule to place a check mark for every taken dose and a prepaid envelope for returning the schedule to confirm adherence. The subjects were advised to return to the paediatric department in case of allergic reactions during the DPT.

Written informed consent to include data in the database was obtained from all subjects or their parents in the case of age $<15$ years.

\section{Patient involvement}

Patients were not directly involved in the design of this study.

\section{Statistical analysis}

Data were expressed either as numbers and percentage or as medians with IQR. Comparisons were made using $\mathrm{X}^{2}$ tests or Fisher's exact tests for categorical variables and Wilcoxon rank sum test for continuous data. $\mathrm{P}<0.05$ was considered to be significant. Statistical analyses were performed using STATA V.16.1.

A sample size of 325 subjects was calculated based on alpha $=0.05$, power $=80 \%$ and anticipated incidence of a positive DPT of $3 \%$ during 1-day challenge and $8 \%$ during 5-day challenge.

\section{RESULTS}

A total of 305 subjects, aged 8 months to 18 years, were included (table 1). None was excluded from the study. The delay from index reaction to DPT was less than 1 year in $133(44 \%)$ of the subjects. A total of $264(86 \%)$ subjects 


\begin{tabular}{|c|c|c|c|}
\hline & N (\%) & Positive & Negative \\
\hline Subjects & 305 & 22 & 283 \\
\hline \multicolumn{4}{|l|}{ Sex } \\
\hline Female & $127(42)$ & $8(36)$ & $119(42)$ \\
\hline Male & $178(58)$ & $14(64)$ & $164(58)$ \\
\hline $\begin{array}{l}\text { Age, years, median } \\
\text { (quartiles) }\end{array}$ & $5.4(2.0 ; 11.4)$ & $4.5(1.8 ; 13.3)$ & $5.5(2.0 ; 11.4$ \\
\hline \multicolumn{4}{|l|}{ Setting } \\
\hline University hospital & $127(42)$ & $6(27)$ & $121(43)$ \\
\hline Regional hospital & $178(58)$ & $16(73)$ & $162(57)$ \\
\hline \multicolumn{4}{|l|}{ Culprit drug } \\
\hline Penicillin V & $122(40)$ & $7(32)$ & $115(41)$ \\
\hline Amoxicillin & $151(50)$ & $13(59)$ & $138(49)$ \\
\hline Dicloxacillin & $17(6)$ & $1(5)$ & $16(6)$ \\
\hline $\begin{array}{l}\text { Amoxicillin+clavulanic } \\
\text { acid }\end{array}$ & $15(5)$ & $1(5)$ & $14(5)$ \\
\hline \multicolumn{4}{|l|}{ Index reaction } \\
\hline Urticaria & $152(49.8)$ & $16(73)$ & $136(48)$ \\
\hline Maculopapular rash & $151(49.5)$ & $6(27)$ & $145(51)$ \\
\hline Erythema multiforme & $2(0.7)$ & $0(0)$ & $2(1)$ \\
\hline \multicolumn{4}{|c|}{ Time from index reaction to DPT } \\
\hline$<1$ year & $133(44)$ & $13(59)$ & $120(42)$ \\
\hline $1-2$ years & $18(6)$ & $2(9)$ & $16(6)$ \\
\hline$>2$ years & $53(17)$ & $3(14)$ & $50(18)$ \\
\hline Unknown & $101(33)$ & $4(18)$ & $97(34)$ \\
\hline \multirow[t]{2}{*}{ Skin prick test } & Positive/negative & & \\
\hline & $0 / 123$ & $0 / 6$ & 0/117 \\
\hline Specific IgE & Positive/negative & & \\
\hline Penicillin V & $13 / 286$ & $1 / 120$ & $12 / 266$ \\
\hline Benzylpenicillin & $9 / 284$ & $1 / 19$ & $8 / 265$ \\
\hline Ampicillin & $8 / 230$ & $1 / 15$ & $7 / 215$ \\
\hline Amoxicillin & $11 / 120$ & $01 / 10 / 20$ & $5 / 110$ \\
\hline
\end{tabular}

DPT, drug provocation test.

returned the challenge schedule confirming adherence with the prolonged DPT.

A total of $22(7 \%)$ of the DPTs were positive (figure 2). The reactions were all non-severe skin rashes either urticaria or maculopapular rash. Three children with urticaria had accompanying mild angioedema and two complained of itching palms. None reacted on the initial 1/10 of a full dose. Three (14\%) of the 22 positive subjects reacted within 1 hour after the first full dose and additional three subjects reacted during the second hour of observation. In all, 12 children reacted before the second full dose was administered. The three children who reacted more than 24 hours after the first full dose reacted at day 3,5 and 6 of the DPT, respectively.

The two children with an index reaction of erythema multiforme both had a negative DPT.

The subjects with a positive DPT did not differ from the negative group with respect to age (median 4.5 vs 5.5 years, $\mathrm{p}=0.75$ ), gender (male: $64 \%$ vs $58 \%, \mathrm{p}=0.60$ ), culprit drug (amoxicillin: $59 \%$ vs $49 \%, \mathrm{p}=0.35$ ) or time

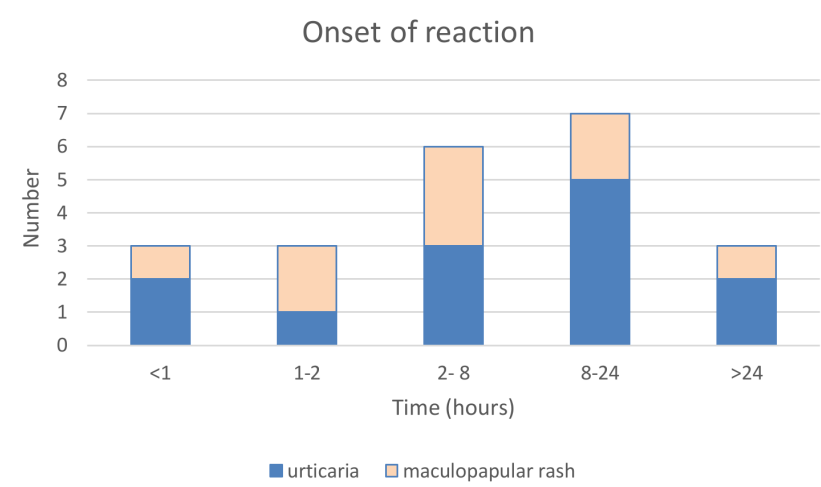

Figure 2 The time interval from first full dose to the appearance of a reaction and the morphology of the rash.

interval from index reaction to DPT (delay $<1$ year: $59 \%$ vs $42 \%$, $\mathrm{p}=0.13$ ).

Only the morphology of the index reaction differed significantly between groups. In $73 \%(\mathrm{n}=16)$ of the positive DPTs, the indication for diagnostic workup was urticaria vs $48 \%(\mathrm{n}=136)$ in the negative group $(\mathrm{p}=0.026)$. Thirteen of the 16 positive children with an urticarial index reaction also had urticaria during the DPT. In all six positive DPTs where the index reaction was a maculopapular rash a similar reaction was observed during the DPT.

The number of positive DPTs did not differ significantly between the two centres (positive DPT: university hospital $5 \%$ vs regional hospital $9 \%, \mathrm{p}=0.16$ ).

All SPTs were negative, while 18 children $(6 \%)$ had at least one positive specific IgE measurement. Of the 41 positive IgE measurements, only nine were $>0.35 \mathrm{kU} / \mathrm{L}$. The highest measured specific IgE was $1.01 \mathrm{kU} / \mathrm{L}$. This subject, a 13-year-old boy, had a negative DPT. Median (quartiles) time from measurement of specific IgE to the DPT was 75 days $(45 ; 132)$. Only one of the children with a positive specific IgE had a positive DPT.

\section{DISCUSSION}

The present study illustrates that the classification of drug hypersensitivity reactions as immediate or nonimmediate reactions poses difficulties. Allergic urticaria is considered as an immediate IgE-mediated reaction ${ }^{5}$ but in case of drug allergy, urticaria can occur several hours or even days after drug consumption. ${ }^{8}$ Likewise, maculopapular rash considered to be a non-immediate reaction ${ }^{6}$ can appear immediately after drug intake. The majority of the parents were not able to recall sufficient details about the index reaction to determine if the reaction appeared within 6 hours of the last administered dose. Consequently, we could only classify the index reaction based on the morphology. As the classification of the rash was based on photographs, history and medical records, an element of incorrectness and recall bias is possible.

The purpose of this study was to investigate if it is necessary to perform extended DPT. Only 6 of the 22 positive reactions appeared during the 2 hours of observation. 
Apparently, at least seven positive reactions (32\%) would have been missed if a second full dose had not been administered after 8 hours. Although, it is possible that some of these subjects would have had a reaction after the first full dose after a time interval of more than 8 hours. While the second full dose seems important, the subsequent 4 days of drug consumption only contributed an additional three (14\%) positive reactions. Mori et al performed 177 five-day provocations in children with suspicion of allergy to amoxicillin. In total, $17(9.6 \%)$ of the DPTs were positive, thereof four on day 5 of the DPT. ${ }^{10}$ These paediatric data differ from the results in adult studies. Fransson et al reported that $47 \%$ and $51 \%$ of the positive reactions in adults appeared after three or more days of prolonged provocation with aminopenicillins and penicillin $\mathrm{V}$, respectively. ${ }^{11}$

Prolonged DPTs thus seems to increase the diagnostic sensitivity. In addition, prolonged DPTs may also increase the proportion of subjects who subsequently ingest penicillin in real-life conditions because parents and physicians are more convinced that the drug will be tolerated. ${ }^{812} 13$

The sensitivity of skin test may be less in children than in adults. ${ }^{14}$ In Denmark, we do not have access to minor and major benzylpenicillin determinants. Consequently, the sensitivity of the SPT is very low. Several authors have found DPT with penicillins without any prior allergy workup to be safe in patients with mild reactions. ${ }^{3} 15-19$ Also, the DAIG Paediatric Task Force suggests that in children with non-immediate mild exanthema a DPT without previous skin test or in vitro testing can be performed. $^{20}$

In the present study, a DPT was performed irrespectively of the specific IgE results. However, all specific IgE levels were low. Therefore, we can only conclude that in the present study, positive specific IgE values between 0.1 $\mathrm{kU} / \mathrm{L}$ and $1.01 \mathrm{kU} / \mathrm{L}$ were not associated with a positive DPT. This is, however, in accordance with the growing evidence that both the sensitivity and the specificity of specific IgE penicillin assays is low. ${ }^{21}$

The main weakness of the study is the time interval from the index reaction to the diagnostic workup. The delay was more than 1 year in approximately half of the subjects. Although this reflects clinical practise at the two Danish centres primarily due to a delay in referral, the sensitivity of the allergy tests decreases over time. ${ }^{22}$ SPT and measurement of specific IgE should ideally be performed 4-6 weeks from the reaction. ${ }^{6}$ Moreover, clinical penicillin tolerance acquisition can occur in both children and adults. ${ }^{23}{ }^{24}$ Therefore, in clinical trials it is important to perform the DPT shortly after the allergy tests. In case of a positive DPT, one should consider to rechallenge the child after a few years as this may reduce the number of children going into adulthood labelled penicillin allergic. Rechallenge, however, may impose a risk of re-sensitisation.

The present study population seems representative for children suspected of being penicillin allergic. The 305 children reside in two different Danish regions, they were consecutively included when referred for penicillin allergy workup and none was excluded.

A DPT with penicillin is a relatively simple and low-risk procedure with important consequences both on an individual and a society level. Due to paucity of data in children, recommendations for adults have been applied. However, children generally have no or only mild reactions in DPTs with penicillin. ${ }^{20}$ SPT has low sensitivity, and we find intradermal tests too painful to be part of the routine workup in children. Consequently, skin tests may be omitted in children with mild reactions. It is likely that it is safe to perform DPTs in children without knowing the specific IgE level. However, we find it necessary to evaluate DPTs in children with a recently measured positive specific IgE to penicillin before a decision to exclude specific IgE-measuring from DPT protocols can be made.

\section{CONCLUSION}

Based on the present study, we suggest that children with mild cutaneous reactions should undergo an in-hospital DPT with penicillin, followed by at least one additional full dose at home. Further studies are needed to investigate the diagnostic value of a positive-specific IgE to penicillin.

Contributors Both authors have contributed to the design of the study, collection of data and drafting of the manuscript. JG conducted the statistical analysis.

Funding The authors have not declared a specific grant for this research from any funding agency in the public, commercial or not-for-profit sectors.

Competing interests None declared.

Patient and public involvement Patients and/or the public were not involved in the design, or conduct, or reporting, or dissemination plans of this research.

Patient consent for publication Not required.

Ethics approval According to The Danish National Committee on Health Research Ethics the study was not subject to notification because all procedures were part of routine medical care and the study did not include any extra diagnostic procedures. Data were prospectively included in a database approved by The Danish Data Protection Agency.

Provenance and peer review Not commissioned; externally peer reviewed.

Data availability statement All data relevant to the study are included in the article or uploaded as online supplementary information.

Open access This is an open access article distributed in accordance with the Creative Commons Attribution Non Commercial (CC BY-NC 4.0) license, which permits others to distribute, remix, adapt, build upon this work non-commercially, and license their derivative works on different terms, provided the original work is properly cited, appropriate credit is given, any changes made indicated, and the use is non-commercial. See: http://creativecommons.org/licenses/by-nc/4.0/.

ORCID iD

Josefine Gradman http://orcid.org/0000-0002-3196-6655

\section{REFERENCES}

1 Erkoçoğlu M, Kaya A, Civelek E, et al. Prevalence of confirmed immediate type drug hypersensitivity reactions among school children. Pediatr Allergy Immunol 2013;24:160-7.

2 Rebelo Gomes E, Fonseca J, Araujo L, et al. Drug allergy claims in children: from self-reporting to confirmed diagnosis. Clin Exp Allergy 2008;38:191-8.

3 Castells M, Khan DA, Phillips EJ. Penicillin allergy. N Engl J Med 2019;381:2338-51.

4 Torres MJ, Celik GE, Whitaker P, et al. A EAACI drug allergy interest group survey on how European allergy specialists deal with $\beta$-lactam allergy. Allergy 2019;74:1052-62. 
5 Torres MJ, Blanca M, Fernandez J, et al. Diagnosis of immediate allergic reactions to beta-lactam antibiotics. Allergy 2003;58:961-72.

6 Romano A, Blanca M, Torres MJ, et al. Diagnosis of nonimmediate reactions to beta-lactam antibiotics. Allergy 2004;59:1153-60.

7 Blanca M, Romano A, Torres MJ, et al. Update on the evaluation of hypersensitivity reactions to betalactams. Allergy 2009;64:183-93.

8 Romano A, Atanaskovic-Markovic M, Barbaud A, et al. Towards a more precise diagnosis of hypersensitivity to beta-lactams - an EAACI position paper. Allergy 2020;75:1300-15.

9 Summaries of product characteristics, 2020. Available: http://www. produktresume.dk/AppBuilder/search

10 Mori F, Cianferoni A, Barni S, et al. Amoxicillin allergy in children: five-day drug provocation test in the diagnosis of nonimmediate reactions. J Allergy Clin Immunol Pract 2015;3:375-80.

11 Fransson S, Mosbech H, Kappel M, et al. The Importance of Prolonged Provocation in Drug Allergy - Results From a Danish Allergy Clinic. J Allergy Clin Immunol Pract 2017;5:1394-401.

12 Ratzon R, Reshef A, Efrati O, et al. Impact of an extended challenge on the effectiveness of $\beta$-lactam hypersensitivity investigation. Ann Allergy Asthma Immunol 2016;116:329-33.

13 Labrosse R, Paradis L, Lacombe-Barrios J, et al. Efficacy and safety of 5-day challenge for the evaluation of nonsevere amoxicillin allergy in children. J Allergy Clin Immunol Pract 2018;6:1673-80.

14 Blanca-López N, Zapatero L, Alonso E, et al. Skin testing and drug provocation in the diagnosis of nonimmediate reactions to aminopenicillins in children. Allergy 2009;64:229-33.

15 Confino-Cohen R, Rosman Y, Meir-Shafrir K, et al. Oral challenge without skin testing safely excludes clinically significant delayedonset penicillin hypersensitivity. J Allergy Clin Immunol Pract 2017;5:669-75.
16 Kuruvilla M, Shih J, Patel K, et al. Direct oral amoxicillin challenge without preliminary skin testing in adult patients with allergy and at low risk with reported penicillin allergy. Allergy Asthma Proc 2019;40:57-61.

17 Mustafa SS, Conn K, Ramsey A. Comparing direct challenge to penicillin skin testing for the outpatient evaluation of penicillin allergy: a randomized controlled trial. J Allergy Clin Immunol Pract 2019;7:2163-70.

18 Wong T, Atkinson A, t'Jong G, et al. Beta-lactam allergy in the paediatric population. Paediatr Child Health 2020;25:62-3.

19 Jaoui A, Delalande D, Siouti S, et al. Safety and cost effectiveness of supervised ambulatory drug provocation tests in children with mild non-immediate reactions to beta-lactams. Allergy 2019;74:2482-4.

20 Gomes ER, Brockow K, Kuyucu S, et al. Drug hypersensitivity in children: report from the pediatric Task force of the EAACl drug allergy interest group. Allergy 2016;71:149-61.

21 van der Poorten M-LM, Van Gasse AL, Hagendorens MM, et al. Serum specific lgE antibodies in immediate drug hypersensitivity. Clin Chim Acta 2020;504:119-24.

22 Fernández TD, Torres MJ, Blanca-López N, et al. Negativization rates of $\mathrm{IgE}$ radioimmunoassay and basophil activation test in immediate reactions to penicillins. Allergy 2009;64:242-8.

23 Tonson la Tour A, Michelet M, Eigenmann PA, et al. Natural history of benign Nonimmediate allergy to beta-lactams in children: a prospective study in Retreated patients after a positive and a negative provocation test. J Allergy Clin Immunol Pract 2018;6:1321-6.

24 Solensky R, Earl HS, Gruchalla RS. Lack of penicillin resensitization in patients with a history of penicillin allergy after receiving repeated penicillin courses. Arch Intern Med 2002;162:822-6. 